voice is recorded and played back to him through earphones with a delay of about half a second, gross disturbances of speech are liable to occur. These disturbances are not unlike those occurring in some forms of dysphasia. Similarly, a recent study carried out in Canada has shown that if sensory stimulation is reduced to a minimum for a period of hours or days, a human subject may experience hallucinations and feelings of unreality which closely resemble those occurring under the influence of hallucinogenic drugs. Similar symptomatology from radically different experimental procedures may or may not mean that a common cerebral mechanism is disturbed; but such converging lines of evidence should, he urged, at least provide strong stimulus for further research.
Malcolm Piercy

\title{
PROGRESS OF CANCER RESEARCH
}

$\mathrm{T}$ HE annual report of the British Empire Cancer Campaign has now expanded in its 1955 edition* to more than 500 pages ; consequently, this short review can only deal with a very limited number of the researches financed and organized by the Campaign. The investigations to be described can be divided into three main groupings, namely, those concerned with carcinogenesis and other studies of that kind, clinico-pathology, and radiology.

In the editorial summary to the report there is a statement which says that "steady progress continues in our understanding of the manner in which cancer-producing substances act". Unfortunately, this happy thought verges on the over-optimistic.

Work on the fashionable cancer problem, the lung cancer-cigarette smoking relationship, continues at a number of centres. One of the main difficulties, from the experimentalist's point of view, is to find some tissue which has the same sensitivity as human lung and upon which the carcinogens present in cigarette smoke could be tested. Sir Ernest Kennaway and co-workers have found $3: 4$ benzpyrene, anthracene, pyrene and acenaphthylene in the condensate from tobacco smoke, and the same compounds have also been detected in smoke from the paper in which the cigarette tobacco is rolled. Sir Ernest has attacked the difficulty of test material by using tissue-culture technique :

"Several workers have demonstrated the carcinogenic power of tobacco condensates upon the skin of the mouse, but neither the species nor the tissue concerned in these experiments is of practical importance. Human tissue ean be used thus only in tissue culture. Dr. Honor B. Fell, of the Strangeways Research Laboratory, Cambridge, has kindly allowed Dr. Ilse Lasnitzki to investigate this matter. Lung tissue obtained from an early human foetus, and therefore sterile, was found to grow well in culture without replacement of epithelium by fibroblasts.

"Such cultures are being exposed to $3: 4$-benzpyrene, and to various fractions of tobacco condensates, in order to learn whether the nuclear changes known to be produced by carcinogens in other tissues can be induced in this instance also."

M. H. Salaman and collaborators have reported interesting developments in the study of the two stages of carcinogenesis ('initiation' and 'promotion'). They find that pre-treatment of mouse skin with urethane (initiation), followed by promotion with croton oil, yields skin tumours; however, urethane, although a highly potent carcinogen for mouse lung, does not induce cancer in mouse skin when applied

* British Empire Cancer Campaign. Thirty-second Annual Report covering the year 1954. Pp. $\mathrm{xxx}+531$. (London: British Empire Cancer Campaign, 1955.) alone. It has now been found that for the initiation stage in mouse skin not only urethane but TEM (trisethyleneimino-3-triazin $\theta$ ) or 1:2-benzanthracene or $\beta$-propiolactone are also effective. The promoter, too (croton oil), can be replaced by iodoacetic acid or by chloracetophenone.

The search for chemotherapeutic drugs for eancer is undergoing an interesting change; less emphasis is now being placed on the therapeutic results achieved, and more and more on the synthesis and preparation of the drugs and upon the mechanism of their cytotoxic action from the points of view of cytology and physical chemistry.

Dr. Percy Stocks, in a study of the influence of social factors on the incidence of breast cancer in women, states:

"The figures suggest that when marriage is delayed the risk of breast cancer is increased, whatever the number of children borne, except in the group married about 30 with long duration of married life ; or, otherwise expressed, that high fertility has a protective effect provided that marriage has not been delayed too long.

"In due course larger numbers will be available to establish this but taking account also of the remarkably low breast cancer rates in Japan where fertility has been high and breast feeding usually very prolonged, sufficient justification has been found for an attempt to seek answers to the following questions :

"(1) Does the mortality excess associated with zero and low fertility indicate simply a greater frequency of breast cancer, or is there also a greater probability of high malignancy when cancer oceurs (i.e. higher fatality without higher inception rates) ?

"(2) Is short duration of breast feeding more important than fow confinements in producing the excess ?"

There are no surprises in the field of morbid anatomy this year. It is good to learn that Bloom's series of cancers of the breast approaches the thousand mark, and that its full statistical analysis is being undertaken. The extraordinary statistical difficulties which bristle about every attempt to assess the effects of different forms of treatment of this disease, and the effect of varying histology, can only be solved by study of really large and uniformly recorded series such as we can hope for in this case. D. H. Patey and A. C. Thackray provide a much-needed study of the whole gland resected in cases of parotid tumour : in none of their cases (save their only adenolymphoma) was there any evidence whatever of multicentric origin-a most important point to be settled in regard to treatment. It is, however, disappointing to find they have used only ten cases.

The review of the tumours of childhood from the Hospital for Sick Children at Great Ormond Street, 
London, continues. There is a valuable collection of sixteen cases, seen over a period of thirty years, of Letterer-Siwe's disease, Hand - Schuller - Christian disease, and eosinophilic granuloma of bone, which demonstrates well the point that all three conditions are examples of a single entity. They range from solitary cured granuloma in older children to rapidly fatal, widely disseminated disease in the infant; but the transitions are too striking to be ignored. The prognosis in these cases seems to be better than is generally supposed. Four cases with solitary lesions were cured by local measures: of six with multiple lesions confined to bone, three are cured and none has died. Even in the disseminated disease there have been two complete cures.

There is also a review from the same source of neuroblastoma, the commonest tumour (except leukæmia) seen at this hospital. They are of especial interest as a base-line for the study of the effects of vitamin $\mathbf{B}_{12}$ on the tumour, first mentioned in last year's report. Of ninety-four cases collected, seventyseven are known to have died, and there have been only two spontaneous regressions. Only twenty cases were regarded as suitable for treatment by surgery or radiotherapy, and of these seven survived more than two years. Nineteen cases in all have now been treated with vitamin $B_{12}$. All were unsuitable for radical conventional treatment, though some have had incomplete surgical or radiation therapy in addition to the $\mathrm{B}_{12}$. Substantial response seems to have been obtained in about half the cases. There has so far been only one really unexceptionable cure, lasting $4 \frac{1}{2}$ years. Two other survivors beyond two years have recurrences ; but several other cases whose follow-up period does not yet justify their being called cures have shown most gratifying immediate responses. It must still be a matter of very great doubt whether we have in $\mathrm{B}_{12}$ a means of cure of neuroblastoma; but it seems probable that we have a means of relief at least as effective as, say, the nitrogen mustards in chronic leukæmia. In any event, the observation throws open a new window in tumour chemotherapy.

Prof. H. N. Green, who is now director of experimental cancer research in the University of Leeds, has combined his interests in carcinogenesis and shock metabolism into a theory of earcinogenesis which has at least the merit of directing attention to some unfamiliar facts. Briefly, his main chain of reasoning is this. Many, perhaps all, chemical carcinogens have an inhibitory effect on the take of transplanted tumours. Some fractions of tar which are not carcinogenic share this inhibitory action with the carcinogens. (Tetraphene is the active agent in some of these extracts.) Use of these non-carcinogens has made it possible to show that the inhibitory action on transplanted tumours is a relatively specific one, not due to any general depression of the host's health ; it has also been shown that there is inhibition of the primary induction of tumours by the carcinogens. The inhibitory action is antagonized by agents, such as cortisone, which depress the immunity to transplanted homologous tissues. These, and other arguments, lead to two hypotheses, of which the first may be readily accepted as possibly true-that the tumour-inhibitory action of carcinogens and noncarcinogens alike is due to a stimulation of the homotransplant immunity reaction. Given this, Prof. Green assumes that the action of these agents must be on the antigenic structure of the cell, and proposes in his second and major hypothesis the view that the essential action of a carcinogen on a cell consists in the alteration of its antigenic structure so that it loses its individuality and becomes antigenically neutral. That loss of antigenic specificity is an important characteristic of some tumour cells -of all transplantable tumours, in factis a proposition that few would deny; that it is a fundamental characteristic of all tumours is harder to accept. But the hypothesis is at least fruitful of exporiments.

There is a tantalizingly brief reference to work in the cytogenetics department at Manchester on the sex chromosomes in transplanted tumours in Microtus, suggesting that the host contributes chromosomes to the tumour cell, of which one hopes to hear more in the future.

The range of the physicist in the realms of cancer research and treatment grows wider and more diverse year by year. Basic physical studies continue, and from them are developed new methods of instru. mentation and measurement. The use of radioactive isotopes is now an everyday matter in a very large number of departments; but work is still going on, and is needed, on increasing the sensitivity and scope of present-day methods of measurement. The construction of automatic devices for the measurement and localization of radioactive material within the human body and on chromatograms is reported from several centres, and the co-operation of the physicist is being sought more and more by his colleagues in their biochemical and biophysical investigations, as well as in the more accustomed rodiotherapeutic uses of radiation.

The commercial development of equipment capable of producing beams of radiation of $1 \mathrm{MeV}$. and greater has necessitated increased study of the radiation effects occurring at these levels, and also the production of methods of measurement more suitable for these energies. A calorimetric method of dosimetry seems to offer a very good alternative to the more standard ionization methods for beams of $\mathrm{X}$-rays of quanta $3 \mathrm{MeV}$. and greater. The use of a 1,000-curie source of cobalt-60 has also led to the study of the dose distribution obtained in the beam, and consideration of the best use of the beam for radiotherapy.

With the increasing use of radioactive materials, and wider range of radiations now in current use, the study of health physics achieves greater prominence each year. In this report, as well as the more usually considered radiation hazards, studies are reported on the possibility of the protection of bronchitics and asthmatics against 'smog', both indoors and out.

At the same centre the radon content of the atmo. sphere under conditions of fog or excessive smoke is being measured with the aid of specially built apparatus which permits the measurement of small amounts of radioactive gases.

The effect of whole-body radiation is being studied at a number of centres with particular reference to protective materials and to the result of partial shielding. The use of iron-59 as an indicator of the effect of various dosage-levels on the erythropoietic system, and also of the effect of changes in dosagerate and of the comparative effect of $\mathrm{X}$ - and neutronirradiation, continues. This should be of considerable value in assessing the total amount of whole radiation received either by design, after radiotherapy, or by chance after an occupational accident or thermonuclear explosion. 